\title{
THE THIRD TERM OF THE LOWER CENTRAL SERIES \\ OF A FREE GROUP AS A SUBGROUP OF THE SECOND
}

Dedicated to the memory of Hanna Neumann

MARTIN WARD

(Received 7 March 1972)

Communicated by M. F. Newman

\section{Introduction}

In this paper the following notation will be used: for any group $G$, positive integer $c$ and non-negative integer $n, G_{c}$ is the $c$ th term of the lower central series of $G$ and $\delta^{n} G_{c}$ is the $n$th term of the derived series of $G_{c}$.

In a free group $F$, the subgroup $F_{2}$ is itself a free group which contains $F_{3}$ as a subgroup. The question arises: how nicely is $F_{3}$ situated in $F_{2}$ ?

In a free metabelian group $G$, the subgroups $G_{2}$ and $G_{3}$ are free abelian and it is easy to see that $G_{3}$ is a direct summand of $G_{2}$, since $G_{2} / G_{3}$ is also free abelian. Returning to the absolutely free group $F$, the simplest analogue of this result, that $F_{3}$ is a free factor of $F_{2}$, is just as easily seen to be false provided that the rank of $F$ is at least 2 , for then $\delta F_{2} \leqq F_{3}$. On the other hand, it is possible to find free generating sets for $F_{2}$ and $F_{3}$ which have a large number of elements in common The question now becomes: how close does $F_{3}$ come to being a free factor of $F_{2}$ ?

To answer this question one looks at members of the free generating set for $F_{3}$ that are not free generators for $F_{2}$ and, as would be expected, these all turn out to lie in $\delta F_{2}$. Suprisingly, they are contained in a free generating set for $\delta F_{2}$ and those members of this free generating set for $\delta F_{2}$ which are not free generators for $F_{3}$ turn out, in turn, to be contained in a free generating set for $\delta F_{3}$. This process continues ad infinitum through free generating sets for the subgroups $\delta^{n} F_{2}$ and $\delta^{n} F_{3}$ and results in the following theorem; the remainder of this paper will be devoted to the details of its proof.

THEOREM. In an absolutely free group $F$ of arbitrary rank there exist subgroups $A_{n}$ and $B_{n}(n=0,1,2, \cdots)$ such that, for each non-negative integer $n$, $\delta^{n} F_{2}$ is the free product of $A_{n}$ and $B_{n}$ and $\delta^{n} F_{3}$ is the free product of $B_{n}$ and $A_{n+1}$. 


\section{Free generating sets for certain subgroups of $F$}

For the remainder of this paper $F$ will be a given absolutely free group of arbitrary rank with a well-ordered free generating set $G$ in terms of which all definitions will be made. A generator of $F$ is to be understood as a member of $G$. The usual notation for commutators in $F$ will be employed: $[x, y]=x^{-1} y^{-1} x y$. The element

$$
\left[b, m_{1} a_{1}, m_{2} a_{2}, \cdots, m_{r} a_{r}\right],
$$

where $r$ and each $m_{i}$ are non-negative integers and $b$ and each $a_{i}$ are members of $F$, is defined inductively. If $r=0$ this is the element $b$, if $r \geqq 1$ and $m_{r}=0$ it is the same as the element

$$
\left[b, m_{1} a_{1}, m_{2} a_{2}, \cdots, m_{r-1} a_{r-1}\right]
$$

and if $r \geqq 1$ and $m_{r} \geqq 1$ it is the element

$$
\left[\left[b, m_{1} a_{1}, m_{2} a_{2}, \cdots, m_{r-1} a_{r-1},\left(m_{r}-1\right) a_{r}\right], a_{r}\right] .
$$

It will be necessary to refer several times to elements of the general form

$$
\left[b, m_{1} a_{1}^{\varepsilon_{1}}, m_{2} a_{2}^{\varepsilon_{2}}, \cdots, m_{r} a_{r}^{\varepsilon_{r}}\right]
$$

where $r$ and each $m_{i}$ are positive integers, each $\varepsilon_{i}= \pm 1, b$ and each $a_{i}$ are members of $F$ which have previously been ordered in some way, $b>a_{1}<a_{2}<\cdots<a_{r}$ and, if $b=\left[b_{1}, b_{2}\right]$ then $b_{2} \leqq a_{1}$. This will be called the standard form and in its various manifestations further restrictions will be placed upon $b$ and the $a_{i}$.

A free generating set for $F_{2}$ was first given by Grunberg in [1], Theorem 5.2 , however it will be necessary to use here the slightly different one given in [2] together with a free generating set for $F_{3}$; these emerge as special cases of Lemma 8 of that paper upon putting $k_{1}=2$ or 3 (for free generating sets for $F_{2}$ or $F_{3}$ respectively) and $k_{i}=2$ for all $i \geqq 2$ in the sequence $K$ of the introduction. For the reader's convenience these free generating sets are described explicitly as the first lemma below. In this lemma, a basic commutator of weight two means as usual an element of the form $[b, a]$ where $a$ and $b$ are generators and $a<b$. Such elements are distinct as written and distinct from the generators. They will be considered to be ordered lexicographically: $\left[b_{1}, a_{1}\right]<\left[b_{2}, a_{2}\right]$ if and only if either $b_{1}<b_{2}$ or $b_{1}=b_{2}$ and $a_{1}<a_{2}$. The orderings of the generators and of the basic commutators of weight two individually are extended to encompass both by specifying that generators always precede commutators.

Lemma 1. (i) The elements of the standard form, where $b$ and each $a_{i}$ are generators, are distinct as written and form a free generating set for $F_{2}$.

(ii) The elements of the standard form, where $b$ is a basic commutator of weight two and each $a_{i}$ is either a generator or a basic commutator of weight two, are distinct as written and form a free generating set for $F_{3}$. 
LEMMA 2. The elements of the form

$$
\left[b_{1}, b_{2}, m_{1} a_{1}^{\varepsilon_{1}}, m_{2} a_{2}^{\ell_{2}}, \cdots, m_{r} a_{r}^{\varepsilon_{r}}\right]
$$

where $r$ is a non-negative integer, each $m_{i}$ is a positive integer, each $\varepsilon_{i}= \pm 1$, $b_{1}, b_{2}$ and each $a_{i}$ are generators and $b_{1}>b_{2} \leqq a_{1}<a_{2}<\cdots<a_{r}$, are distinct as written and form a free generating set for $F_{2}$.

Note that elements of the form (1) may alternatively be described as elements of the standard form except that $r$ may possibly be zero, where $b$ is a basic commutator of weight two and each $a_{i}$ is a generator.

Proof of Lemma 2. (I am indebted to Professor Gruenberg for suggesting this proof, which in much more satisfactory than my own.) Let $S$ be the free generating set for $F_{2}$ given in Lemma 1 (i) and let $T$ be the putative free generating set for $F_{2}$ given in the statement of Lemma 2. An endomorphism $\theta$ of $F_{2}$ is now defined by prescribing its action on $S$. Let

$$
x=\left[b_{1}, m_{1} a_{1}^{\varepsilon_{1}}, m_{2} a_{2}^{\varepsilon_{2}}, \cdots, m_{r} a_{r}^{\varepsilon_{r}}\right]
$$

be an arbitrary member of $S$; then set

$$
\begin{aligned}
x \theta & =x & & \text { if } \varepsilon_{1}=+1 \\
& =\left[b_{1}, a_{1}, m_{1} a_{1}^{-1}, m_{2} a_{2}^{\varepsilon_{2}}, \cdots, m_{r} a_{r}^{\varepsilon_{r}}\right] & & \text { if } \varepsilon_{1}=-1
\end{aligned} .
$$

It is easy to see that $\theta$ maps $S$ one-to-one onto $T$. It is now sufficient to show that that $\theta$ is an automorphism of $F_{2}$.

We now prove, by induction over $m$, that for any $m \geqq 1,\left[b, a, m a^{-1}\right]$ can be written as a product of elements of the forms $[b, a]$ and $\left[b, l a^{-1}\right](1 \leqq l \leqq m)$ in which the factor $\left[b, m a^{-1}\right]$ appears exactly once and that with exponent \pm 1 . The initial case $(m=1)$ is verified by exhibiting the well known law

$$
\left[b, a, a^{-1}\right]=[b, a]^{-1}\left[b, a^{-1}\right]^{-1} \text {. }
$$

Assuming now that $\left[b, a, m a^{-1}\right]$ can be written in the required form, consider

$$
\begin{aligned}
{\left[b, a,(m+1) a^{-1}\right] } & =\left[b, a, m a^{-1}, a^{-1}\right] \\
& =\left[b, a, m a^{-1}\right]^{-1}\left[b, a, m a^{-1}\right]^{a-1} .
\end{aligned}
$$

The first term here, by the inductive hypothesis, can be written as a product of elements of the forms $[b, a]$ and $\left[b, l a^{-1}\right](1 \leqq l \leqq m)$, all of which are of the required form; note that $\left[b,(m+1) a^{-1}\right]$ does not appear as a factor in this product. The second term can be written as another such product conjugated by $a^{-1}$ and thus as a product of erements of the forms $[b, a]^{a^{-1}}$ and $\left[b, l a^{-1}\right]^{a^{-1}}$ $(1 \leqq l \leqq m)$ in which the factor $\left[b, m a^{-1}\right]^{a^{-1}}$ appears exactly once and that with exponent \pm 1 . Since

and

$$
[b, a]^{a-1}=\left[b, a^{-1}\right]^{-1}
$$




$$
\left[b, l a^{-1}\right]^{a^{-1}}=\left[b, l a^{-1}\right]\left[b,(l+1) a^{-1}\right]
$$

the statement is proved.

A similar argument now shows, this time by induction over $r$, that for any $m \geqq 1$ and $r \geqq 0$,

$$
\left[b, a, m a^{-1}, c_{1}, c_{2}, \cdots, c_{r}\right]
$$

can be written as a product of elements of the forms

$$
\left[b, a, c_{1}, c_{2}, \cdots, c_{q}\right] \quad(0 \leqq q \leqq r)
$$

and

$$
\left[b, l a^{-1}, c_{1}, c_{2}, \cdots, c_{q}\right] \quad(0 \leqq q \leqq r, 1 \leqq l \leqq m)
$$

in which the factor $\left[b, m a^{-1}, c_{1}, c_{2}, \cdots, c_{r}\right]$ appears exactly once and that with exponent \pm 1 .

Let us consider $S$ to be well-ordered in such a way as to preserve weight and so that

$$
\left[b, m_{1} a_{1}^{+1}, m_{2} a_{2}^{\varepsilon_{2}}, \cdots, m_{r} a_{r}^{\varepsilon_{r}}\right]<\left[b, m_{1} a_{1}^{-1}, m_{2} a_{2}^{\varepsilon_{2}}, \cdots, m_{r} a_{r}^{\varepsilon_{r}}\right] .
$$

Then $S$ is a well-ordered free generating set for $F_{2}$ and $\theta$ is an endomorphism of $F_{2}$ with the property that, for each member $x$ of $S, x \theta$ can be written as a product of members of $S$ none of which exceed $x$ itself and which contains $x$ as a factor exactly once and that with exponent \pm 1 : if $x$ is of the form (2) and $\varepsilon_{1}=+1$, then $x \theta=x$ and this result is trivially true. If $\varepsilon_{1}=-1$ then $x \theta$ is given by (3) and the result has been established by the second inductive proof above.

It follows that $\theta$ is an automorphism of $F_{2}$, as required.

LEMMA 3. Let $H$ be a subgroup of $F$ with a well-ordered free generating set which is the disjoint union of two subsets $X$ and $Y$ such that every element of $X$ precedes every element of $Y$. Let $X^{\prime}$ be the set of all elements of the standard form with $b \in X \cup Y$ and each $a_{i} \in X$ and let $Y^{\prime}$ be the set of all elements of the standard form with $b \in Y \cup X^{\prime}$ and each $a_{i} \in Y$. Then the members of $X^{\prime} \cup Y^{\prime}$ are distinct as written and form a free generating set for the derived group of $H$.

Proof. Writing $Z$ for the set of all elements of the standard form where $b$ and each $a_{i}$ belong to $X \cup Y$, it follows from Lemma 1 (i) that the elements of $Z$ are distinct as written and freely generate the derived group of $H$; it remains to prove that $X^{\prime} \cup Y^{\prime}=Z$.

An element $x$ of $X^{\prime} \cup Y^{\prime}$ is of the standard form

$$
x=\left[b, m_{1} a_{1}^{\varepsilon_{1}}, m_{2} a_{2}^{\varepsilon_{2}}, \cdots, m_{r} a_{r}^{\varepsilon_{r}}\right]
$$

where either $b \in X \cup Y$ and each $a_{i} \in X$ or $b \in Y \cup X^{\prime}$ and each $a_{i} \in Y$. Then $x$ is obviously a member of $Z$ unless $b \in X^{\prime}$ and each $a_{i} \in Y$; but then $b$ is itself of standard form, 


$$
b=\left[d, n_{1} c_{1}^{\delta_{1}}, n_{2} c_{2}^{\delta_{2}}, \cdots, n_{s} c_{s}^{\delta_{s}}\right]
$$

say, where $d \in X \cup Y$ and each $c_{i} \in X$. Thus

$$
x=\left[d, n_{1} c_{1}^{\delta_{1}}, n_{2} c_{2}^{\delta_{2}}, \cdots, n_{s} c_{s}^{\delta_{s}}, m_{1} a_{1}^{\varepsilon_{1}}, m_{2} a_{2}^{\varepsilon_{2}}, \cdots, m_{r} a_{r}^{\varepsilon_{r}}\right]
$$

and, since $c_{s} \in X$ and $a_{1} \in Y, c_{s}<a_{1}$ so this is also standard form and again $x \in Z$. This proves that $X^{\prime} \cup Y^{\prime} \subseteq Z$. Conversely, any element $x$ of $z$ is of the standard form (4) where $b$ and each $b_{i}$ belong to $X \cup Y$. If all the $a_{i}$ belong to $X$ then $x \in X^{\prime}$ and if all the $b_{i}$ belong to $Y$ then so does $b$, since $b>a_{1}$, and thus $x \in Y^{\prime}$. It remains to consider the case where some of the $a_{i}$ belong to $X$ and some belong to $Y$. Since members of $X$ precede those of $Y$, there is an integer $k$ $(1 \leqq k<r)$ such that $a_{1}, a_{2}, \cdots, a_{k} \in X$ and $a_{k+1}, a_{k+2}, \cdots, a_{r} \in Y$. Then writing

$$
c=\left[b, m_{1} a_{1}^{\varepsilon_{1}}, m_{2} a_{2}^{\varepsilon_{2}}, \cdots, m_{k} a_{k}^{\varepsilon_{k}}\right],
$$

$c \in X^{\prime}$ and

$$
x=\left[c, m_{k+1} a_{k+1}^{\varepsilon_{k+1}}, m_{k+2} a_{k+2}^{\varepsilon_{k+2}}, \cdots, m_{r} a_{r}^{\varepsilon_{r}}\right]
$$

is thus a member of $Y^{\prime}$. Hence $Z \subseteq X^{\prime} \cup Y^{\prime}$ and the lemma is proved.

\section{Proof of the theorem}

Let $X_{0}$ be the subset of $F$ consisting of all basic commutators of weight two, that is, all elements of the form (1) with $r=0$; let $Y_{0}$ be the subset of $F$ consisting of all elements of the form (1) with $r \geqq 1$. By Lemma 2, the elements of $X_{0} \cup Y_{0}$ are distinct as written and freely generate $F_{2}$; we will assume them to be wellordered in any way which preserves weight. Under this order, elements of $X_{0}$ precede those of $Y_{0}$.

Subsets $X_{n}$ and $Y_{n}$ of $F$ are now defined inductively for all positive integers $n$ : assuming that the subsets $X_{n-1}$ and $Y_{n-1}$ have already been defined and wellordered in such a way that element of $X_{n-1}$ precede those of $Y_{n-1}$ and that the members of $X_{n-1} \cup Y_{n-1}$ are distinct as written and freely generate $\delta^{n-1} F_{2}$, let $X_{n}$ be the set of all elements of the standard form with $b \in X_{n-1} \cup Y_{n-1}$ and each $a_{i} \in X_{n-1}$ and let $Y_{n}$ be the set of all elements of the standard form with $b \in Y_{n-1} \cup X_{n}$ and each $a_{i} \in Y_{n-1}$. It follows from Lemma 3 that the elements of $X_{n} \cup Y_{n}$ are distinct as written and freely generate $\delta^{n} F_{2}$. Well-order $X_{n} \cup Y_{n}$ in any way such that elements of $X_{n}$ precede those of $Y_{n}$.

Defining, for each non-negative integer $n, A_{n}$ and $B_{n}$ to be the subgroups of $F$ generated by $X_{n}$ and $Y_{n}$ respectively, it follows that $\delta^{n} F_{2}$ is the free product of $A_{n}$ and $B_{n}$.

It is now clear that, in the union of all the sets $X_{n} \cup Y_{n}$, elements are distinct as written. The orders defined, for each individual $n$, on the sets $X_{n} \cup Y_{n}$ may now be extended to their union by specifying that whenever $m<n$, elements of $X_{m} \cup Y_{m}$ 
precede those of $X_{n} \cup Y_{n}$. In particular, elements of $B_{n}$ precede those of $A_{n+1}$.

An argument similar to the proof of Lemma 3 yields the fact that the free generating set for $F_{3}$ given in Lemma 1 (i) is just $Y_{0} \cup X_{1}$. Lemma 3 then shows by induction that, for any non-negative integer $n, \delta^{n} F_{3}$ is freely generated by $Y_{n} \cup X_{n+1}$. It follows that $\delta^{n} F_{3}$ is the free product of $B_{n}$ and $A_{n+1}$.

\section{References}

[1] K. W. Gruenberg, 'Residual Properties of Infinite Soluble Groups', Proc. London Math. Soc. Ser. 3.7 (1957), 29-62.

[2] Martin Ward, 'Bases for Polynilpotent Groups', Proc. London Math. Soc. Ser. (3) 24 (1972), 409-431.

York University

4700 Keele Street

Downsview 463

Ontario, Canada

Present address

Department of Pure Mathematics

Australian National University

Canberra 